\title{
Search for Resonant Diphoton Production with the D0 Detector
}

V. M. Abazov, ${ }^{36}$ B. Abbott,${ }^{75}$ M. Abolins, ${ }^{65}$ B. S. Acharya, ${ }^{29}$ M. Adams, ${ }^{51}$ T. Adams,${ }^{49}$ E. Aguilo, ${ }^{6}$ M. Ahsan, ${ }^{59}$ G. D. Alexeev, ${ }^{36}$ G. Alkhazov, ${ }^{40}$ A. Alton, ${ }^{64, *}$ G. Alverson, ${ }^{63}$ G. A. Alves, ${ }^{2}$ M. Anastasoaie, ${ }^{35}$ L. S. Ancu, ${ }^{35}$ T. Andeen, ${ }^{53}$ B. Andrieu, ${ }^{17}$ M. S. Anzelc, ${ }^{53}$ M. Aoki,${ }^{50}$ Y. Arnoud, ${ }^{14}$ M. Arov,${ }^{60}$ M. Arthaud, ${ }^{18}$ A. Askew, ${ }^{49, \dagger}$ B. Åsman,${ }^{41}$ A.C. S. Assis Jesus, ${ }^{3}$ O. Atramentov, ${ }^{49}$ C. Avila,${ }^{8}$ J. BackusMayes,${ }^{82}$ F. Badaud,${ }^{13}$ L. Bagby, ${ }^{50}$ B. Baldin,${ }^{50}$ D. V. Bandurin, ${ }^{59}$ P. Banerjee, ${ }^{29}$ S. Banerjee,${ }^{29}$ E. Barberis, ${ }^{63}$ A.-F. Barfuss, ${ }^{15}$ P. Bargassa, ${ }^{80}$ P. Baringer,${ }^{58}$ J. Barreto, ${ }^{2}$ J. F. Bartlett ${ }^{50}$ U. Bassler, ${ }^{18}$ D. Bauer, ${ }^{43}$ S. Beale, ${ }^{6}$ A. Bean,${ }^{58}$ M. Begalli, ${ }^{3}$ M. Begel,${ }^{73}$ C. Belanger-Champagne, ${ }^{41}$ L. Bellantoni, ${ }^{50}$ A. Bellavance, ${ }^{50}$ J. A. Benitez, ${ }^{65}$ S. B. Beri, ${ }^{27}$ G. Bernardi,${ }^{17}$ R. Bernhard,${ }^{23}$ I. Bertram, ${ }^{42}$ M. Besançon, ${ }^{18}$ R. Beuselinck, ${ }^{43}$ V. A. Bezzubov,${ }^{39}$ P. C. Bhat,${ }^{50}$ V. Bhatnagar ${ }^{27}$ G. Blazey,${ }^{52}$ F. Blekman, ${ }^{43}$ S. Blessing, ${ }^{49}$ K. Bloom, ${ }^{67}$ A. Boehnlein, ${ }^{50}$ D. Boline, ${ }^{62}$ T. A. Bolton, ${ }^{59}$ E. E. Boos,${ }^{38}$ G. Borissov, ${ }^{42}$ T. Bose, ${ }^{77}$ A. Brandt ${ }^{78}$ R. Brock, ${ }^{65}$ G. Brooijmans, ${ }^{70}$ A. Bross ${ }^{50}$ D. Brown, ${ }^{19}$ X. B. Bu, ${ }^{7}$ N. J. Buchanan, ${ }^{49}$ D. Buchholz,${ }^{53}$ M. Buehler, ${ }^{81}$ V. Buescher, ${ }^{22}$ V. Bunichev, ${ }^{38}$ S. Burdin, ${ }^{42,}$ T. H. Burnett ${ }^{82}$ C. P. Buszello, ${ }^{43}$ P. Calfayan, ${ }^{25}$ B. Calpas, ${ }^{15}$ S. Calvet, ${ }^{16}$ J. Cammin,${ }^{71}$ M. A. Carrasco-Lizarraga, ${ }^{33}$ E. Carrera, ${ }^{49}$ W. Carvalho, ${ }^{3}$ B. C. K. Casey, ${ }^{50}$ H. Castilla-Valdez, ${ }^{33}$ S. Chakrabarti, ${ }^{72}$ D. Chakraborty, ${ }^{52}$ K. M. Chan, ${ }^{55}$ A. Chandra, ${ }^{48}$ E. Cheu, ${ }^{45}$ D. K. Cho, ${ }^{62}$ S. Choi, ${ }^{32}$ B. Choudhary, ${ }^{28}$ L. Christofek, ${ }^{77}$ T. Christoudias, ${ }^{43}$ S. Cihangir, ${ }^{50}$ D. Claes,${ }^{67}$ J. Clutter, ${ }^{58}$ M. Cooke, ${ }^{50}$ W. E. Cooper, ${ }^{50}$ M. Corcoran, ${ }^{80}$ F. Couderc,${ }^{18}$ M.-C. Cousinou, ${ }^{15}$ S. Crépé-Renaudin,,${ }^{14}$ V. Cuplov,${ }^{59}$ D. Cutts, ${ }^{77}$ M. Ćwiok, ${ }^{30}$ H. da Motta, ${ }^{2}$ A. Das, ${ }^{45}$ G. Davies, ${ }^{43}$ K. De,${ }^{78}$ S. J. de Jong, ${ }^{35}$ E. De La Cruz-Burelo, ${ }^{33}$ C. De Oliveira Martins, ${ }^{3}$ K. DeVaughan,${ }^{67}$ F. Déliot,${ }^{18}$ M. Demarteau ${ }^{50}$ R. Demina, ${ }^{71}$ D. Denisov ${ }^{50}$ S. P. Denisov, ${ }^{39}$ S. Desai,${ }^{50}$ H. T. Diehl,${ }^{50}$ M. Diesburg, ${ }^{50}$ A. Dominguez,${ }^{67}$ T. Dorland, ${ }^{82}$ A. Dubey, ${ }^{28}$ L. V. Dudko, ${ }^{38}$ L. Duflot, ${ }^{16}$ S. R. Dugad,${ }^{29}$ D. Duggan, ${ }^{49}$ A. Duperrin, ${ }^{15}$ S. Dutt, ${ }^{27}$ J. Dyer, ${ }^{65}$ A. Dyshkant,${ }^{52}$ M. Eads, ${ }^{67}$ D. Edmunds, ${ }^{65}$ J. Ellison, ${ }^{48}$ V. D. Elvira, ${ }^{50}$ Y. Enari, ${ }^{77}$ S. Eno, ${ }^{61}$ P. Ermolov, ${ }^{38,} \S$ M. Escalier, ${ }^{15}$ H. Evans,${ }^{54}$ A. Evdokimov, ${ }^{73}$ V. N. Evdokimov, ${ }^{39}$ A. V. Ferapontov, ${ }^{59}$ T. Ferbel,,${ }^{61,71}$ F. Fiedler, ${ }^{24}$ F. Filthaut, ${ }^{35}$ W. Fisher, ${ }^{50}$ H. E. Fisk,${ }^{50}$ M. Fortner, ${ }^{52}$ H. Fox ${ }^{42}$ S. Fu, ${ }^{50}$ S. Fuess, ${ }^{50}$ T. Gadfort, ${ }^{70}$ C. F. Galea, ${ }^{35}$ C. Garcia, ${ }^{71}$ A. Garcia-Bellido, ${ }^{71}$ V. Gavrilov, ${ }^{37}$ P. Gay, ${ }^{13}$ W. Geist, ${ }^{19}$ W. Geng, ${ }^{15,65}$ C. E. Gerber ${ }^{51}$ Y. Gershtein, ${ }^{49, \dagger}$ D. Gillberg, ${ }^{6}$ G. Ginther, ${ }^{71}$ B. Gómez, ${ }^{8}$ A. Goussiou, ${ }^{82}$

P. D. Grannis, ${ }^{72}$ H. Greenlee, ${ }^{50}$ Z. D. Greenwood ${ }^{60}$ E. M. Gregores,${ }^{4}$ G. Grenier,${ }^{20}$ Ph. Gris, ${ }^{13}$ J.-F. Grivaz, ${ }^{16}$

A. Grohsjean, ${ }^{25}$ S. Grünendahl, ${ }^{50}$ M. W. Grünewald, ${ }^{30}$ F. Guo, ${ }^{72}$ J. Guo, ${ }^{72}$ G. Gutierrez, ${ }^{50}$ P. Gutierrez, ${ }^{75}$ A. Haas,${ }^{70}$

N. J. Hadley, ${ }^{61}$ P. Haefner, ${ }^{25}$ S. Hagopian, ${ }^{49}$ J. Haley ${ }^{68}$ I. Hall, ${ }^{65}$ R. E. Hall,${ }^{47}$ L. Han, ${ }^{7}$ K. Harder,${ }^{44}$ A. Harel,${ }^{71}$ J. M. Hauptman, ${ }^{57}$ J. Hays, ${ }^{43}$ T. Hebbeker, ${ }^{21}$ D. Hedin, ${ }^{52}$ J. G. Hegeman, ${ }^{34}$ A. P. Heinson, ${ }^{48}$ U. Heintz, ${ }^{62}$ C. Hensel, ${ }^{22,8}$ K. Herner, ${ }^{72}$ G. Hesketh, ${ }^{63}$ M. D. Hildreth, ${ }^{55}$ R. Hirosky, ${ }^{81}$ T. Hoang, ${ }^{49}$ J. D. Hobbs ${ }^{72}$ B. Hoeneisen, ${ }^{12}$ M. Hohlfeld, ${ }^{22}$ S. Hossain, ${ }^{75}$ P. Houben, ${ }^{34}$ Y. Hu, ${ }^{72}$ Z. Hubacek,,${ }^{10}$ N. Huske, ${ }^{17}$ V. Hynek, ${ }^{9}$ I. Iashvili, ${ }^{69}$ R. Illingworth,${ }^{50}$ A. S. Ito, ${ }^{50}$ S. Jabeen, ${ }^{62}$ M. Jaffré, ${ }^{16}$ S. Jain, ${ }^{75}$ K. Jakobs, ${ }^{23}$ C. Jarvis, ${ }^{61}$ R. Jesik ${ }^{43}$ K. Johns,${ }^{45}$ C. Johnson, ${ }^{70}$ M. Johnson, ${ }^{50}$ D. Johnston, ${ }^{67}$ A. Jonckheere, ${ }^{50}$ P. Jonsson, ${ }^{43}$ A. Juste, ${ }^{50}$ E. Kajfasz, ${ }^{15}$ D. Karmanov, ${ }^{38}$ P. A. Kasper, ${ }^{50}$ I. Katsanos, ${ }^{70}$ V. Kaushik, ${ }^{78}$ R. Kehoe ${ }^{79}$ S. Kermiche, ${ }^{15}$ N. Khalatyan, ${ }^{50}$ A. Khanov ${ }^{76}$ A. Kharchilava, ${ }^{69}$ Y. N. Kharzheev, ${ }^{36}$ D. Khatidze, ${ }^{70}$ T. J. Kim, ${ }^{31}$ M. H. Kirby, ${ }^{53}$ M. Kirsch, ${ }^{21}$ B. Klima, ${ }^{50}$ J. M. Kohli, ${ }^{27}$ J.-P. Konrath, ${ }^{23}$ A. V. Kozelov, ${ }^{39}$

J. Kraus, ${ }^{65}$ T. Kuhl, ${ }^{24}$ A. Kumar, ${ }^{69}$ A. Kupco, ${ }^{11}$ T. Kurča,${ }^{20}$ V. A. Kuzmin, ${ }^{38}$ J. Kvita, ${ }^{9}$ F. Lacroix, ${ }^{13}$ D. Lam, ${ }^{55}$ S. Lammers, ${ }^{70}$ G. Landsberg, ${ }^{77}$ P. Lebrun, ${ }^{20}$ W. M. Lee, ${ }^{50}$ A. Leflat, ${ }^{38}$ J. Lellouch, ${ }^{17}$ J. Li $,{ }^{78,8 \S} \mathrm{L} . \mathrm{Li},{ }^{48}$ Q. Z. Li,${ }^{50}$ S. M. Lietti, ${ }^{5}$ J. K. Lim, ${ }^{31}$ J. G. R. Lima, ${ }^{52}$ D. Lincoln,${ }^{50}$ J. Linnemann,${ }^{65}$ V. V. Lipaev, ${ }^{39}$ R. Lipton, ${ }^{50}$ Y. Liu, ${ }^{7}$ Z. Liu, ${ }^{6}$ A. Lobodenko, ${ }^{40}$ M. Lokajicek, ${ }^{11}$ P. Love, ${ }^{42}$ H. J. Lubatti, ${ }^{82}$ R. Luna-Garcia, ${ }^{33, \|}$ A. L. Lyon, ${ }^{50}$ A. K. A. Maciel, ${ }^{2}$ D. Mackin, ${ }^{80}$ R. J. Madaras, ${ }^{46}$ P. Mättig, ${ }^{26}$ A. Magerkurth, ${ }^{64}$ P. K. Mal, ${ }^{82}$ H. B. Malbouisson, ${ }^{3}$ S. Malik, ${ }^{67}$ V. L. Malyshev, ${ }^{36}$ Y. Maravin, ${ }^{59}$ B. Martin, ${ }^{14}$ R. McCarthy, ${ }^{72}$ M. M. Meijer, ${ }^{35}$ A. Melnitchouk, ${ }^{66}$ L. Mendoza, ${ }^{8}$ P. G. Mercadante, ${ }^{5}$ M. Merkin, ${ }^{38}$ K. W. Merritt, ${ }^{50}$ A. Meyer, ${ }^{21}$ J. Meyer, ${ }^{22,8}$ J. Mitrevski, ${ }^{70}$ R. K. Mommsen,${ }^{44}$ N. K. Mondal, ${ }^{29}$ R. W. Moore, ${ }^{6}$ T. Moulik, ${ }^{58}$ G. S. Muanza, ${ }^{15}$ M. Mulhearn, ${ }^{70}$ O. Mundal, ${ }^{22}$ L. Mundim, ${ }^{3}$ E. Nagy, ${ }^{15}$ M. Naimuddin,${ }^{50}$ M. Narain, ${ }^{77}$ H. A. Neal, ${ }^{64}$ J. P. Negret ${ }^{8}$ P. Neustroev, ${ }^{40}$ H. Nilsen, ${ }^{23}$ H. Nogima, ${ }^{3}$ S. F. Novaes, ${ }^{5}$ T. Nunnemann, ${ }^{25}$ D. C. O'Neil, ${ }^{6}$ G. Obrant,${ }^{40}$ C. Ochando, ${ }^{16}$ D. Onoprienko, ${ }^{59}$ N. Oshima, ${ }^{50}$ N. Osman, ${ }^{43}$ J. Osta, ${ }^{55}$ R. Otec, ${ }^{10}$ G. J. Otero y Garzón, ${ }^{1}$

M. Owen ${ }^{44}$ M. Padilla, ${ }^{48}$ P. Padley, ${ }^{80}$ M. Pangilinan, ${ }^{77}$ N. Parashar, ${ }^{56}$ S.-J. Park,${ }^{22,8}$ S. K. Park, ${ }^{31}$ J. Parsons, ${ }^{70}$

R. Partridge,${ }^{77}$ N. Parua,${ }^{54}$ A. Patwa,${ }^{73}$ G. Pawloski ${ }^{80}$ B. Penning,${ }^{23}$ M. Perfilov,${ }^{38}$ K. Peters,${ }^{44}$ Y. Peters,${ }^{26}$ P. Pétroff, ${ }^{16}$ M. Petteni, ${ }^{43}$ R. Piegaia, ${ }^{1}$ J. Piper, ${ }^{65}$ M.-A. Pleier ${ }^{22}$ P. L. M. Podesta-Lerma,${ }^{33, \pi l}$ V. M. Podstavkov, ${ }^{50}$ Y. Pogorelov, ${ }^{55}$ M.-E. Pol, ${ }^{2}$ P. Polozov, ${ }^{37}$ B. G. Pope,${ }^{65}$ A. V. Popov,${ }^{39}$ C. Potter,${ }^{6}$ W. L. Prado da Silva, ${ }^{3}$ H. B. Prosper, ${ }^{49}$ S. Protopopescu,${ }^{73}$ J. Qian, ${ }^{64}$ A. Quadt, ${ }^{22,8}$ B. Quinn, ${ }^{66}$ A. Rakitine, ${ }^{42}$ M. S. Rangel, ${ }^{2}$ K. Ranjan ${ }^{28}$ P. N. Ratoff, ${ }^{42}$ P. Renkel ${ }^{79}$ P. Rich, ${ }^{44}$ M. Rijssenbeek ${ }^{72}$ I. Ripp-Baudot, ${ }^{19}$ F. Rizatdinova,${ }^{76}$ S. Robinson, ${ }^{43}$ R. F. Rodrigues, ${ }^{3}$ M. Rominsky, ${ }^{75}$ C. Royon, ${ }^{18}$ 
P. Rubinov, ${ }^{50}$ R. Ruchti, ${ }^{55}$ G. Safronov,${ }^{37}$ G. Sajot,${ }^{14}$ A. Sánchez-Hernández,${ }^{33}$ M. P. Sanders, ${ }^{17}$ B. Sanghi,${ }^{50}$ G. Savage, ${ }^{50}$

L. Sawyer, ${ }^{60}$ T. Scanlon, ${ }^{43}$ D. Schaile, ${ }^{25}$ R. D. Schamberger ${ }^{72}$ Y. Scheglov, ${ }^{40}$ H. Schellman, ${ }^{53}$ T. Schliephake, ${ }^{26}$

S. Schlobohm, ${ }^{82}$ C. Schwanenberger, ${ }^{44}$ R. Schwienhorst ${ }^{65}$ J. Sekaric, ${ }^{49}$ H. Severini,${ }^{75}$ E. Shabalina,${ }^{51}$ M. Shamim, ${ }^{59}$ V. Shary, ${ }^{18}$ A. A. Shchukin, ${ }^{39}$ R. K. Shivpuri, ${ }^{28}$ V. Siccardi,${ }^{19}$ V. Simak, ${ }^{10}$ V. Sirotenko, ${ }^{50}$ P. Skubic, ${ }^{75}$ P. Slattery, ${ }^{71}$ D. Smirnov, ${ }^{55}$ G. R. Snow, ${ }^{67}$ J. Snow, ${ }^{74}$ S. Snyder, ${ }^{73}$ S. Söldner-Rembold ${ }^{44}$ L. Sonnenschein, ${ }^{17}$ A. Sopczak ${ }^{42}$

M. Sosebee, ${ }^{78}$ K. Soustruznik, ${ }^{9}$ B. Spurlock, ${ }^{78}$ J. Stark, ${ }^{14}$ V. Stolin, ${ }^{37}$ D. A. Stoyanova, ${ }^{39}$ J. Strandberg, ${ }^{64}$ S. Strandberg, ${ }^{41}$ M. A. Strang, ${ }^{69}$ E. Strauss, ${ }^{72}$ M. Strauss, ${ }^{75}$ R. Ströhmer, ${ }^{25}$ D. Strom,${ }^{53}$ L. Stutte,${ }^{50}$ S. Sumowidagdo, ${ }^{49}$ P. Svoisky, ${ }^{35}$ A. Sznajder, ${ }^{3}$ A. Tanasijczuk, ${ }^{1}$ W. Taylor, ${ }^{6}$ B. Tiller, ${ }^{25}$ F. Tissandier, ${ }^{13}$ M. Titov, ${ }^{18}$ V. V. Tokmenin,${ }^{36}$ I. Torchiani, ${ }^{23}$ D. Tsybychev, ${ }^{72}$ B. Tuchming, ${ }^{18}$ C. Tully, ${ }^{68}$ P. M. Tuts, ${ }^{70}$ R. Unalan, ${ }^{65}$ L. Uvarov, ${ }^{40}$ S. Uvarov,${ }^{40}$ S. Uzunyan,${ }^{52}$ B. Vachon, ${ }^{6}$ P. J. van den Berg, ${ }^{34}$ R. Van Kooten,${ }^{54}$ W. M. van Leeuwen, ${ }^{34}$ N. Varelas, ${ }^{51}$ E. W. Varnes, ${ }^{45}$ I. A. Vasilyev, ${ }^{39}$ P. Verdier, ${ }^{20}$ L. S. Vertogradov, ${ }^{36} \mathrm{M}$. Verzocchi, ${ }^{50} \mathrm{D}$. Vilanova, ${ }^{18} \mathrm{~F}$. Villeneuve-Seguier, ${ }^{43} \mathrm{P}$. Vint, ${ }^{43}$ P. Vokac, ${ }^{10} \mathrm{M}$. Voutilainen, ${ }^{67, * *}$ R. Wagner, ${ }^{68}$ H. D. Wahl, ${ }^{49}$ M. H. L. S. Wang, ${ }^{50}$ J. Warchol, ${ }^{55}$ G. Watts, ${ }^{82}$ M. Wayne, ${ }^{55}$ G. Weber, ${ }^{24}$ M. Weber, ${ }^{50, \dagger \dagger}$ L. Welty-Rieger, ${ }^{54}$ A. Wenger, ${ }^{23,+1}$ N. Wermes,${ }^{22}$ M. Wetstein, ${ }^{61}$ A. White, ${ }^{78}$ D. Wicke, ${ }^{26}$ M. R. J. Williams,${ }^{42}$ G. W. Wilson, ${ }^{58}$ S. J. Wimpenny, ${ }^{48}$ M. Wobisch, ${ }^{60}$ D. R. Wood, ${ }^{63}$ T. R. Wyatt, ${ }^{44}$ Y. Xie, ${ }^{77}$ C. Xu, ${ }^{64}$ S. Yacoob, ${ }^{53}$ R. Yamada, ${ }^{50}$ W.-C. Yang, ${ }^{44}$ T. Yasuda,${ }^{50}$ Y. A. Yatsunenko, ${ }^{36}$ Z. Ye,${ }^{50}$ H. Yin, ${ }^{7}$ K. Yip, ${ }^{73}$ H. D. Yoo, ${ }^{77}$ S. W. Youn, ${ }^{53}$ J. Yu, ${ }^{78}$ C. Zeitnitz, ${ }^{26}$ S. Zelitch, ${ }^{81}$ T. Zhao, ${ }^{82}$ B. Zhou, ${ }^{64}$ J. Zhu, ${ }^{72}$ M. Zielinski, ${ }^{71}$ D. Zieminska, ${ }^{54}$ L. Zivkovic ${ }^{70}$ V. Zutshi, ${ }^{52}$ and E. G. Zverev ${ }^{38}$

\title{
(D0 Collaboration)
}

\author{
${ }^{1}$ Universidad de Buenos Aires, Buenos Aires, Argentina \\ ${ }^{2}$ LAFEX, Centro Brasileiro de Pesquisas Físicas, Rio de Janeiro, Brazil \\ ${ }^{3}$ Universidade do Estado do Rio de Janeiro, Rio de Janeiro, Brazil \\ ${ }^{4}$ Universidade Federal do ABC, Santo André, Brazil \\ ${ }^{5}$ Instituto de Física Teórica, Universidade Estadual Paulista, São Paulo, Brazil \\ ${ }^{6}$ University of Alberta, Edmonton, Alberta, Canada, \\ Simon Fraser University, Burnaby, British Columbia, Canada, \\ York University, Toronto, Ontario, Canada, \\ and McGill University, Montreal, Quebec, Canada \\ ${ }^{7}$ University of Science and Technology of China, Hefei, People's Republic of China \\ ${ }^{8}$ Universidad de los Andes, Bogotá, Colombia \\ ${ }^{9}$ Center for Particle Physics, Charles University, Prague, Czech Republic \\ ${ }^{10}$ Czech Technical University, Prague, Czech Republic \\ ${ }^{11}$ Center for Particle Physics, Institute of Physics, Academy of Sciences of the Czech Republic, Prague, Czech Republic \\ ${ }^{12}$ Universidad San Francisco de Quito, Quito, Ecuador \\ ${ }^{13}$ LPC, Université Blaise Pascal, CNRS/IN2P3, Clermont, France \\ ${ }^{14}$ LPSC, Université Joseph Fourier Grenoble 1, CNRS/IN2P3, Institut National Polytechnique de Grenoble, Grenoble, France \\ ${ }^{15}$ CPPM, Aix-Marseille Université, CNRS/IN2P3, Marseille, France \\ ${ }^{16}$ LAL, Université Paris-Sud, IN2P3/CNRS, Orsay, France \\ ${ }^{17}$ LPNHE, IN2P3/CNRS, Universités Paris VI and VII, Paris, France \\ ${ }^{18}$ CEA, Irfu, SPP, Saclay, France \\ ${ }^{19}$ IPHC, Université Louis Pasteur, CNRS/IN2P3, Strasbourg, France \\ ${ }^{20} I P N L$, Université Lyon 1, CNRS/IN2P3, Villeurbanne, France and Université de Lyon, Lyon, France \\ ${ }^{21}$ III. Physikalisches Institut A, RWTH Aachen University, Aachen, Germany \\ ${ }^{22}$ Physikalisches Institut, Universität Bonn, Bonn, Germany \\ ${ }^{23}$ Physikalisches Institut, Universität Freiburg, Freiburg, Germany \\ ${ }^{24}$ Institut für Physik, Universität Mainz, Mainz, Germany \\ ${ }^{25}$ Ludwig-Maximilians-Universität München, München, Germany \\ ${ }^{26}$ Fachbereich Physik, University of Wuppertal, Wuppertal, Germany \\ ${ }^{27}$ Panjab University, Chandigarh, India \\ ${ }^{28}$ Delhi University, Delhi, India \\ ${ }^{29}$ Tata Institute of Fundamental Research, Mumbai, India \\ ${ }^{30}$ University College Dublin, Dublin, Ireland \\ ${ }^{31}$ Korea Detector Laboratory, Korea University, Seoul, Korea \\ ${ }^{32}$ SungKyunKwan University, Suwon, Korea \\ ${ }^{33}$ CINVESTAV, Mexico City, Mexico \\ ${ }^{34}$ FOM-Institute NIKHEF and University of Amsterdam/NIKHEF, Amsterdam, The Netherlands
}


${ }^{35}$ Radboud University Nijmegen/NIKHEF, Nijmegen, The Netherlands

${ }^{36}$ Joint Institute for Nuclear Research, Dubna, Russia

${ }^{37}$ Institute for Theoretical and Experimental Physics, Moscow, Russia

${ }^{38}$ Moscow State University, Moscow, Russia

${ }^{39}$ Institute for High Energy Physics, Protvino, Russia

${ }^{40}$ Petersburg Nuclear Physics Institute, St. Petersburg, Russia

${ }^{41}$ Lund University, Lund, Sweden,

Royal Institute of Technology and Stockholm University, Stockholm, Sweden, and Uppsala University, Uppsala, Sweden

${ }^{42}$ Lancaster University, Lancaster, United Kingdom

${ }^{43}$ Imperial College, London, United Kingdom

${ }^{44}$ University of Manchester, Manchester, United Kingdom

${ }^{45}$ University of Arizona, Tucson, Arizona 85721, USA

${ }^{46}$ Lawrence Berkeley National Laboratory and University of California, Berkeley, California 94720, USA

${ }^{47}$ California State University, Fresno, California 93740, USA

${ }^{48}$ University of California, Riverside, California 92521, USA

${ }^{49}$ Florida State University, Tallahassee, Florida 32306, USA

${ }^{50}$ Fermi National Accelerator Laboratory, Batavia, Illinois 60510, USA

${ }^{51}$ University of Illinois at Chicago, Chicago, Illinois 60607, USA

${ }^{52}$ Northern Illinois University, DeKalb, Illinois 60115, USA

${ }^{53}$ Northwestern University, Evanston, Illinois 60208, USA

${ }^{54}$ Indiana University, Bloomington, Indiana 47405, USA

${ }^{55}$ University of Notre Dame, Notre Dame, Indiana 46556, USA

${ }^{56}$ Purdue University Calumet, Hammond, Indiana 46323, USA

${ }^{57}$ Iowa State University, Ames, Iowa 50011, USA

${ }^{58}$ University of Kansas, Lawrence, Kansas 66045, USA

${ }^{59}$ Kansas State University, Manhattan, Kansas 66506, USA

${ }^{60}$ Louisiana Tech University, Ruston, Louisiana 71272, USA

${ }^{61}$ University of Maryland, College Park, Maryland 20742, USA

${ }^{62}$ Boston University, Boston, Massachusetts 02215, USA

${ }^{63}$ Northeastern University, Boston, Massachusetts 02115, USA

${ }^{64}$ University of Michigan, Ann Arbor, Michigan 48109, USA

${ }^{65}$ Michigan State University, East Lansing, Michigan 48824, USA

${ }^{66}$ University of Mississippi, University, Mississippi 38677, USA

${ }^{67}$ University of Nebraska, Lincoln, Nebraska 68588, USA

${ }^{68}$ Princeton University, Princeton, New Jersey 08544, USA

${ }^{69}$ State University of New York, Buffalo, New York 14260, USA

${ }^{70}$ Columbia University, New York, New York 10027, USA

${ }^{71}$ University of Rochester, Rochester, New York 14627, USA

${ }^{72}$ State University of New York, Stony Brook, New York 11794, USA

${ }^{73}$ Brookhaven National Laboratory, Upton, New York 11973, USA

${ }^{74}$ Langston University, Langston, Oklahoma 73050, USA

${ }^{75}$ University of Oklahoma, Norman, Oklahoma 73019, USA

${ }^{76}$ Oklahoma State University, Stillwater, Oklahoma 74078, USA

${ }^{77}$ Brown University, Providence, Rhode Island 02912, USA

${ }^{78}$ University of Texas, Arlington, Texas 76019, USA

${ }^{79}$ Southern Methodist University, Dallas, Texas 75275, USA

${ }^{80}$ Rice University, Houston, Texas 77005, USA

${ }^{81}$ University of Virginia, Charlottesville, Virginia 22901, USA

${ }^{82}$ University of Washington, Seattle, Washington 98195, USA

(Received 14 January 2009; published 12 June 2009)

We present a search for a narrow resonance in the inclusive diphoton final state using $\sim 2.7 \mathrm{fb}^{-1}$ of data collected with the D0 detector at the Fermilab Tevatron $p \bar{p}$ Collider. We observe good agreement between the data and the background prediction, and set the first 95\% C.L. upper limits on the production cross section times the branching ratio for decay into a pair of photons for resonance masses between 100 and $150 \mathrm{GeV}$. This search is also interpreted in the context of several models of electroweak symmetry breaking with a Higgs boson decaying into two photons.

DOI: 10.1103/PhysRevLett.102.231801

PACS numbers: $14.80 . \mathrm{Bn}, 13.85 . \mathrm{Qk}, 13.85 . \mathrm{Rm}$ 
At a hadron collider, diphoton $(\gamma \gamma)$ production allows detailed studies of the standard model (SM) [1], as well as searches for new phenomena, such as new heavy resonances [2], extra spatial dimensions, or cascade decays of heavy new particles [3]. Within the SM, continuum $\gamma \gamma+$ $X$ production is characterized by a steeply falling $\gamma \gamma$ mass $\left(M_{\gamma \gamma}\right)$ spectrum, on top of which a heavy resonance decaying into $\gamma \gamma$ can potentially be observed. In particular, this is considered one of the most promising discovery channels for a light SM Higgs boson at the LHC [4], despite the small branching ratio of $B(H \rightarrow \gamma \gamma) \sim 0.2 \%$ for $110<$ $M_{H}<140 \mathrm{GeV}[5,6]$. At the Tevatron, the dominant SM Higgs boson production mechanism is gluon fusion (GF) $(g g \rightarrow H)$, followed by associated production with a $W$ or $Z$ boson $\left(q \bar{q}^{\prime} \rightarrow V H, V=W, Z\right)$, and vector boson fusion (VBF) $(V V \rightarrow H)$ [7-9]. While the SM Higgs production rate at the Tevatron is not sufficient to observe it in the $\gamma \gamma$ mode, the $H g g$ and $H \gamma \gamma$ couplings, being loop-mediated, are particularly sensitive to new physics effects. Furthermore, in some models beyond the SM [2], $B(H \rightarrow$ $\gamma \gamma)$ can be enhanced significantly relative to the SM prediction.

In this Letter, we present a search for a narrow resonance in the $M_{\gamma \gamma}$ spectrum using a data sample collected by the D0 detector [10] at the Fermilab Tevatron Collider. The selection of an inclusive $\gamma \gamma+X$ sample and the use of the $M_{\gamma \gamma}$ spectrum make the results of this search quasi-model independent. We use the SM Higgs boson $\left(H_{\mathrm{SM}}\right)$ with $H \rightarrow$ $\gamma \gamma$ as a reference model, resulting in the first such search at the Tevatron, and a forerunner to similar planned searches at the LHC. Additionally, we consider other models of electroweak symmetry breaking (EWSB) with enhanced $B(H \rightarrow \gamma \gamma)$ [2], as a consequence of suppressed Higgs couplings to either (i) all fermions (known as bosonic or fermiophobic Higgs boson, $H_{f}$ ), (ii) only down-type fermions ( $H_{u}$, as it gives mass to up-type fermions), or (iii) only top and bottom quarks (known as electroweak Higgs boson, $\left.H_{\mathrm{EW}}\right)$. All models considered have SM-like production cross sections, with the exception of the $H_{f}$ and $H_{\text {EW }}$ models, where GF is absent or has a negligibly small cross section.

The subdetectors most relevant to this analysis are the central tracking system, composed of a silicon microstrip tracker (SMT) and a central fiber tracker (CFT) embedded in a $2 \mathrm{~T}$ solenoidal magnetic field, the central preshower (CPS), and the liquid-argon and uranium sampling calorimeter. The CPS is located immediately before the inner layer of the calorimeter and is formed of one radiation length of absorber followed by several layers of scintillating strips. The calorimeter consists of a central section with coverage of $|\eta|<1.1$ [11], and two end calorimeters covering up to $|\eta| \simeq 4$.2. The electromagnetic (EM) section of the calorimeter is segmented into four longitudinal layers $(\mathrm{EM} i, i=1,4)$ with transverse segmentation of $\Delta \eta \times$ $\Delta \phi=0.1 \times 0.1$ [11], except in EM3, where it is $0.05 \times$
0.05 . The calorimeter is well suited for a precise measurement of electron and photon energies, providing a resolution of $\sim 3.6 \%$ at energies of $\sim 50 \mathrm{GeV}$. The data used in this analysis were collected using triggers requiring at least two clusters of energy in the EM calorimeter and correspond to an integrated luminosity of $2.7 \pm 0.2 \mathrm{fb}^{-1}$ [12].

Events are selected by requiring at least two photon candidates with transverse momentum $p_{T}>25 \mathrm{GeV}$ and $|\eta|<1.1$, for which the trigger requirements are fully efficient. The photon $p_{T}$ is computed with respect to the reconstructed event primary vertex (PV) with the highest number of associated tracks, which is required to be within $60 \mathrm{~cm}$ of the geometrical center of the detector along the beam axis. The PV reconstruction efficiency in $\gamma \gamma+X$ events is $\sim 98 \%$, with $\sim 95 \%$ probability to match the true vertex. Photons are selected from EM clusters reconstructed within a cone with radius $\mathcal{R}=$ $\sqrt{(\Delta \eta)^{2}+(\Delta \phi)^{2}}=0.2$ by requiring (i) $\geq 97 \%$ of the cluster energy is deposited in the EM calorimeter, (ii) the calorimeter isolation variable $I=\left[E_{\mathrm{tot}}(0.4)-\right.$ $\left.E_{\mathrm{EM}}(0.2)\right] / E_{\mathrm{EM}}(0.2)<0.1$, where $E_{\mathrm{tot}}(\mathcal{R})\left(E_{\mathrm{EM}}(\mathcal{R})\right)$ is the total (EM) energy in a cone of radius $\mathcal{R}$, (iii) the energy-weighted shower width in the $r-\phi$ plane in EM3 is $<\sqrt{14} \mathrm{~cm}$, and (iv) the scalar sum of the $p_{T}$ of all tracks $\left(p_{T \text { trk }}^{\text {sum }}\right)$ originating from the primary vertex in an annulus of $0.05<\mathcal{R}<0.4$ around the cluster is $<2 \mathrm{GeV}$. To suppress electrons misidentified as photons, the EM clusters are required to not be spatially matched to tracker activity, either a reconstructed track, or a density of hits in the SMT and CFT consistent with that of an electron [13]. To suppress jets misidentified as photons, a neural network (NN) is trained using a set of variables sensitive to differences between photons and jets in the tracker activity and in the energy deposits in the calorimeter and CPS: $p_{T \text { trk }}^{\text {sum }}$, the numbers of cells above a threshold in EM1 within $\mathcal{R}<0.2$ and $0.2<\mathcal{R}<0.4$ of the EM cluster, the number of CPS clusters within $\mathcal{R}<0.1$ of the EM cluster, and the squared-energy-weighted width of the energy deposit in the CPS. The NN is trained using $\gamma \gamma$ and dijet Monte Carlo (MC) samples and its performance is verified using a data sample of $Z \rightarrow \ell^{+} \ell^{-} \gamma(\ell=e, \mu)$ events. Figure 1(a) compares the NN output $\left(O_{\mathrm{NN}}\right)$ spectrum for photons and jets. Photon candidates are required to have $O_{\mathrm{NN}}>0.1$, which is $\sim 98 \%$ efficient for real photons and rejects $\sim 50 \%$ of misidentified jets. Finally, $M_{\gamma \gamma}$, computed from the two highest $p_{T}$ photons, is required to be $>60 \mathrm{GeV}$. In total, 5608 events are selected in data.

All MC samples used in this analysis are generated using PYTHIA [14] with CTEQ6L [15] parton distribution functions (PDFs), and processed through a GEANT-based [16] simulation of the D0 detector and the same reconstruction software as the data. Signal samples are generated separately for GF, VH, and VBF production and normalized using the theoretical cross sections [7-9] and branching ratio predictions from HDECAY [5]. 

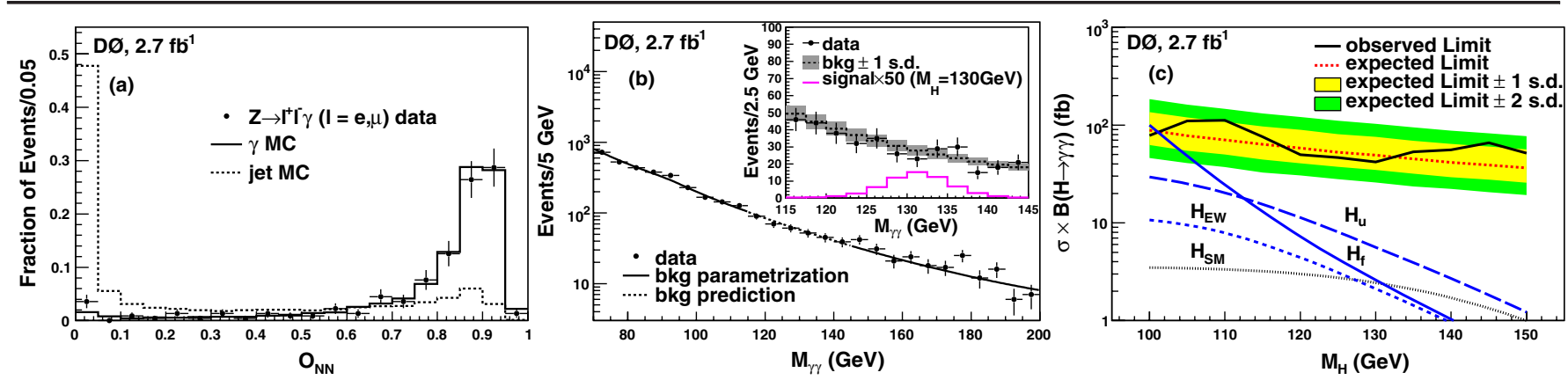

FIG. 1 (color online). (a) Normalized $O_{\mathrm{NN}}$ spectrum for photons and jets. (b) $M_{\gamma \gamma}$ spectrum in data (points) compared to the total background parametrization (solid line), including the DDP contribution derived via a sideband fit, and the total background prediction (dashed line) inside the search region for $M_{H}=130 \mathrm{GeV}$. The inset figure compares the data to the total background prediction inside the search region including its 1 standard deviation (s.d.) uncertainty band, as well as the expected $H_{\mathrm{SM}}$ signal scaled by a factor of 50 . (c) Observed and expected 95\% C.L. upper limits on $\sigma \times B$ as a function of $M_{H}$. Also shown are the predictions for $\sigma \times B$ in the different EWSB scenarios discussed in the text.

This analysis is affected by instrumental backgrounds such as $\gamma+$ jet, dijet, and $Z / \gamma^{*} \rightarrow e^{+} e^{-}$(ZDY) production, with jets or electrons misidentified as photons, as well as an irreducible background from direct diphoton production (DDP). All backgrounds, except for ZDY, are estimated directly from data.

The ZDY background is estimated using the MC simulation, normalized to the next-to-next-to-leading-order cross section [17]. The selection efficiencies determined by the $\mathrm{MC}$ simulation are corrected to the corresponding values measured in the data. On average each electron has a $2 \%$ probability to satisfy the photon selection criteria, mainly due to the inefficiency of the track-match veto requirements. The total contribution from ZDY is estimated to be $88 \pm 10$ events.

Backgrounds due to $\gamma+$ jet and dijet events are directly estimated from data by using a $4 \times 4$ matrix background estimation method [18]. After final event selection, a tightened $O_{\mathrm{NN}}$ requirement $\left(O_{\mathrm{NN}}>0.75\right)$ is used to classify the events into four categories depending on whether the two highest- $p_{T}$ photons, only the leading photon, only the trailing photon, or neither of the two photons satisfy this requirement. The corresponding numbers of events, after subtraction of the estimated ZDY contributions, are denoted as $N_{p p}, N_{p f}, N_{f p}$, and $N_{f f}$. The different relative efficiency of the $O_{\mathrm{NN}}>$ 0.75 requirement between real photons and jets allows the estimation of the sample composition by solving a linear system of equations: $\left(N_{p p}, N_{p f}, N_{f p}, N_{f f}\right)^{T}=$ $\mathcal{E}\left(N_{\gamma \gamma}, N_{\gamma j}, N_{j \gamma}, N_{j j}\right)^{T}$, where $N_{\gamma \gamma}\left(N_{j j}\right)$ is the number of $\gamma \gamma$ (dijet) events and $N_{\gamma j}\left(N_{j \gamma}\right)$ is the number of $\gamma+$ jet events with the leading (trailing) cluster as the photon. The $4 \times 4$ matrix $\mathcal{E}$ contains the efficiency terms (parametrized as a function of $|\eta|$ ), estimated in photon and jet MC samples and validated in data. The estimated sample composition is $N_{\gamma \gamma}=3155 \pm 125$ (stat), $N_{\gamma j+j \gamma}=1680 \pm$ 149(stat), and $N_{j j}=685 \pm 93$ (stat). The shape of the $M_{\gamma \gamma}$ spectrum for the sum of the $\gamma+$ jet and dijet backgrounds is obtained from an independent control data sample by requiring $O_{\mathrm{NN}}<0.1$ for one of the photon candidates, and is parametrized with an exponential function. The resulting shape is found to be in excellent agreement with that derived by directly applying the $4 \times 4$ matrix method bin by bin in the final selected sample, but has smaller statistical fluctuations, especially in the high $M_{\gamma \gamma}$ region.

TABLE I. Numbers of selected events in data, expected backgrounds, expected $H_{\mathrm{SM}}$ signal, and signal acceptance (for each production mechanism: GF, VH, VBF), in the search region for different $M_{H}$ values. The expected signal includes contributions from $\mathrm{GF}, \mathrm{VH}$, and $\mathrm{VBF}$ processes, the latter two representing $\sim 21 \%-24 \%$ of the total signal.

\begin{tabular}{lcccccc}
\hline \hline$M_{H}(\mathrm{GeV})$ & 100 & 110 & 120 & 130 & 140 & \multicolumn{1}{c}{150} \\
\hline$Z / \gamma^{*} \rightarrow e^{+} e^{-}$ & $55 \pm 7$ & $17 \pm 3$ & $6 \pm 2$ & $5 \pm 1$ & $4 \pm 1$ & 1 \\
$\gamma \gamma$ & $742 \pm 62$ & $481 \pm 42$ & $324 \pm 34$ & $236 \pm 30$ & $161 \pm 28$ & $124 \pm 22$ \\
$\gamma j+j j$ & $540 \pm 66$ & $319 \pm 39$ & $204 \pm 25$ & $133 \pm 16$ & $89 \pm 11$ & $61 \pm 8$ \\
Total background & $1337 \pm 29$ & $817 \pm 26$ & $534 \pm 19$ & $374 \pm 12$ & $254 \pm 7$ & $188 \pm 5$ \\
Data & 1385 & 827 & 544 & 357 & 270 & 202 \\
$H_{\text {SM }}$ signal & $1.62 \pm 0.11$ & $1.61 \pm 0.11$ & $1.51 \pm 0.10$ & $1.26 \pm 0.08$ & $0.90 \pm 0.06$ & $0.54 \pm 0.04$ \\
Acceptance $(\%)$ & $19.9,18.8,20.3$ & $20.4,19.9,21.6$ & $21.0,20.6,22.3$ & $21.5,21.2,22.9$ & $21.8,22.0,23.5$ & $22.1,22.2,24.1$ \\
\hline \hline
\end{tabular}


After subtraction of the ZDY, $\gamma+$ jet, and dijet background contributions, the $M_{\gamma \gamma}$ spectrum is examined for the presence of a narrow resonance. For each assumed $M_{H}$ value (between 100 and $150 \mathrm{GeV}$, in steps of $5 \mathrm{GeV}$ ), the search region is defined to be $\left(M_{H}-15 \mathrm{GeV}, M_{H}+\right.$ $15 \mathrm{GeV}$ ), where $15 \mathrm{GeV}$ corresponds to about five times the expected $M_{\gamma \gamma}$ resolution. The DDP background is estimated by performing a sideband fit to the $M_{\gamma \gamma}$ spectrum in the 70 to $200 \mathrm{GeV}$ range (this excludes the search region) using an exponential function [see Fig. 1(b)]. Such a parametrization has been validated using a next-to-leading-order calculation for this process [19].

Systematic uncertainties affecting the normalization and shape of the $M_{\gamma \gamma}$ spectrum are estimated for both signal and backgrounds. Uncertainties affecting the ZDY background normalization include integrated luminosity (6.1\%), electron misidentification rate $(14.3 \%)$, and ZDY cross section $(3.9 \%)$. Such uncertainties are propagated, via the $4 \times 4$ matrix method, to the estimated normalization of the $\gamma+$ jet and dijet background contributions, affected in addition by the uncertainty on the $O_{\mathrm{NN}}>0.75$ selection efficiency for photons $(2 \%)$ and jets $(10 \%)$. The uncertainty in the shape of the $\gamma+$ jet and dijet $M_{\gamma \gamma}$ spectrum is given by the statistics of the control data sample used to parametrize it. The above uncertainties, as well as the statistical uncertainties of the sideband fitting method, result in systematic uncertainties in the normalization and shape of the DDP background contribution. Uncertainties affecting the signal normalization include integrated luminosity (6.1\%), acceptance due to the photon identification efficiency (6.8\%), and PDFs (1.7\%-2.2\%) [15]. Finally, the location of the peak in the $M_{\gamma \gamma}$ spectrum for signal is affected by the uncertainty in the relative datato-MC photon energy scale $(0.6 \%)$.

Table I shows the number of events in the data, expected background, and expected $H_{\mathrm{SM}}$ signal in six different search regions. The inset in Fig. 1(b) illustrates the $M_{\gamma \gamma}$ spectrum in the search region for $M_{H}=130 \mathrm{GeV}$, found to be in good agreement with the background prediction. The $M_{\gamma \gamma}$ spectrum in the search region is used to derive upper limits on the production cross section times branching ratio for $H \rightarrow \gamma \gamma(\sigma \times B)$ as a function of $M_{H}$. The SM prediction for the ratio of the production cross sections for the three signal production mechanisms is assumed. Limits are calculated at the 95\% C.L. using the modified frequentist approach with a Poisson log-likelihood ratio test statistic $[20,21]$. The impact of systematic uncertainties is incorporated via convolution of the Poisson probability distributions for signal and background with Gaussian distributions corresponding to the different sources of systematic uncertainty. The correlations in systematic uncertainties are maintained between signal and backgrounds.

The resulting limits on $\sigma \times B$ are given in Table II, and displayed in Fig. 1(c). Although the SM Higgs boson is
TABLE II. Observed and expected 95\% C.L. upper limits on $\sigma \times B$ (in fb) for different $M_{H}$ values. The expected limit is defined as the median of the distribution of limits in backgroundonly pseudoexperiments.

$\begin{array}{llllllllllll}M_{H}(\mathrm{GeV}) & 100 & 105 & 110 & 115 & 120 & 125 & 130 & 135 & 140 & 145 & 150\end{array}$

\begin{tabular}{llllllllllll}
\hline Exp. $\sigma \times B$ & 88 & 78 & 71 & 63 & 58 & 53 & 49 & 45 & 41 & 39 & 36
\end{tabular}

\begin{tabular}{llllllllllll} 
Obs. $\sigma \times B$ & 78 & 110 & 112 & 76 & 50 & 46 & 42 & 53 & 56 & 66 & 52 \\
\hline
\end{tabular}

used as a reference model, the fact that the signal acceptance is found to be almost independent of the production mechanism (see Table I) makes the estimated limits applicable to other models of new physics with a narrow resonance decaying into $\gamma \gamma$. In the context of models of EWSB with enhanced $B(H \rightarrow \gamma \gamma)$, the current search excludes a $H_{f}$ boson with $M_{H}<101 \mathrm{GeV}$ at $95 \%$ C.L., improving (slightly) upon previous results at the Tevatron [22]. While none of the other EWSB scenarios explored can currently be excluded, the expected sensitivity is within less than a factor of 4 of the prediction for the $H_{u}$ model for $M_{H}<$ $110 \mathrm{GeV}$, and only a factor $\sim 20$ above the SM prediction for $115 \leq M_{H} \leq 130 \mathrm{GeV}$. As a result, this search contributes to the overall sensitivity of the SM Higgs boson search at the Tevatron from the combination of multiple channels [23]. Assuming the same integrated luminosity in all channels and a single Tevatron experiment, this analysis is expected to improve the combined upper limit on the SM Higgs production cross section by $\sim 5 \%$ for $115 \leq M_{H} \leq$ $130 \mathrm{GeV}$. Finally, this search is used to derive $95 \%$ C.L. upper limits on $B(H \rightarrow \gamma \gamma)$ between $14.1 \%$ and $33.9 \%$ for $M_{H}$ in the range $100-150 \mathrm{GeV}$, in the case of models where the Higgs boson does not couple to the top quark. Conversely, for models where the GF production mode is available, this inclusive search allows improvement of the upper limits on $B(H \rightarrow \gamma \gamma)$ to $3.4 \%-7.2 \%$ in the same mass range. These represent the most stringent limits on $B(H \rightarrow \gamma \gamma)$ for $M_{H}$ in the range $100-150 \mathrm{GeV}$ to date, significantly improving upon previous results from LEP and the Tevatron [22].

In summary, we have performed an inclusive search for a narrow resonance with mass between 100 and $150 \mathrm{GeV}$ decaying into $\gamma \gamma$ at the Tevatron. This channel is used to increase the overall sensitivity of the SM Higgs boson search program at the Tevatron [23] and allows the probe of new physics models predicting an enhanced rate for $H \rightarrow \gamma \gamma$.

We thank the staffs at Fermilab and collaborating institutions, and acknowledge support from the DOE and NSF (U.S.); CEA and CNRS/IN2P3 (France); FASI, Rosatom and RFBR (Russia); CNPq, FAPERJ, FAPESP and FUNDUNESP (Brazil); DAE and DST (India); Colciencias (Colombia); CONACyT (Mexico); KRF and KOSEF (Korea); CONICET and UBACyT (Argentina); FOM (The Netherlands); STFC (United Kingdom); MSMT and GACR (Czech Republic); CRC Program, 
CFI, NSERC and WestGrid Project (Canada); BMBF and DFG (Germany); SFI (Ireland); The Swedish Research Council (Sweden); CAS and CNSF (China); and the Alexander von Humboldt Foundation (Germany).

*Visitor from Augustana College, Sioux Falls, SD, USA. ${ }^{\dagger}$ Visitor from Rutgers University, Piscataway, NJ, USA.

${ }^{\ddagger}$ Visitor from The University of Liverpool, Liverpool, UK.

${ }^{\S}$ Visitor from II. Physikalisches Institut, Georg-AugustUniversity, Göttingen, Germany.

"Visitor from Centro de Investigacion en ComputacionIPN, Mexico City, Mexico.

${ }^{\text {II }}$ Visitor from ECFM, Universidad Autonoma de Sinaloa, Culiacán, Mexico.

**Visitor from Helsinki Institute of Physics, Helsinki, Finland.

${ }^{\dagger}$ Visitor from Universität Bern, Bern, Switzerland.

Visitor from Universität Zürich, Zürich, Switzerland.

$\S \S$ Deceased.

[1] C. Balazs, E. L. Berger, P. Nadolsky, and C.-P. Yuan, Phys. Rev. D 76, 013009 (2007), and references therein.

[2] S. Mrenna and J. Wells, Phys. Rev. D 63, 015006 (2000), and references therein.

[3] See, e.g., G. F. Giudice and R. Rattazzi, Phys. Rep. 322, 419 (1999); M. C. Kumar, P. Mathews, V. Ravindran, and A. Tripathi, Phys. Lett. B 672, 45 (2009), and references therein.

[4] G. Aad et al. (ATLAS Collaboration), arXiv:0901.0512; G. L. Bayatian et al. (CMS Collaboration), J. Phys. G 34, 995 (2007).
[5] A. Djouadi, J. Kalinowski, and M. Spira, Comput. Phys. Commun. 108, 56 (1998).

[6] Throughout this Letter we adopt units where $c=1$.

[7] S. Catani et al., J. High Energy Phys. 07 (2003) 028.

[8] U. Aglietti et al., arXiv:hep-ph/0610033.

[9] K. A. Assamagan et al., arXiv:hep-ph/0406152.

[10] V. M. Abazov et al. (D0 Collaboration), Nucl. Instrum. Methods Phys. Res., Sect. A 565, 463 (2006).

[11] Pseudorapidity is defined as $\eta=-\ln [\tan (\theta / 2)]$, where $\theta$ is the polar angle relative to the proton beam direction. $\phi$ is defined as the azimuthal angle in the plane transverse to the proton beam direction.

[12] T. Andeen et al., Report No. FERMILAB-TM-2365, 2007.

[13] V. M. Abazov et al. (D0 Collaboration), Phys. Lett. B 659, 856 (2008).

[14] T. Sjöstrand et al., Comput. Phys. Commun. 135, 238 (2001); we use PYTHIA version v6.323.

[15] J. Pumplin et al., J. High Energy Phys. 07 (2002) 012; D. Stump et al., J. High Energy Phys. 10 (2003) 046.

[16] R. Brun and F. Carminati, CERN Program Library Long Writeup Report No. W5013, 1993; we use GEANT version v3.21.

[17] R. Hamberg, W. L. van Neerven, and T. Matsuura, Nucl. Phys. B359, 343 (1991); 644, 403 (2002).

[18] D. Acosta et al. (CDF Collaboration), Phys. Rev. Lett. 95, 022003 (2005).

[19] T. Binoth et al., Eur. Phys. J. C 16, 311 (2000).

[20] T. Junk, Nucl. Instrum. Methods Phys. Res., Sect. A 434, 435 (1999); A. Read, CERN Report No. 2000-005, 2000.

[21] W. Fisher, Report No. FERMILAB-TM-2386-E, 2006.

[22] V. M. Abazov et al. (D0 Collaboration), Phys. Rev. Lett. 101, 051801 (2008), and references therein.

[23] TEVNPH Working Group, for the CDF and D0 Collaborations, arXiv:0903.4001. 